\title{
Immobilization of Nanobodies with Vapor-Deposited Polymer Encapsulation for Robust Biosensors
}

\author{
$\ddagger$ Ruolan Fan ${ }^{1}, \ddagger$ Jiale Du ${ }^{1}, \ddagger$ Kwang-Won Park $^{1}$, Lin Hui Chang ${ }^{1}$, Eric R. Strieter ${ }^{*}$, Trisha L. Andrew ${ }^{1,2 *}$ \\ ${ }^{1}$ Department of Chemistry, University of Massachusetts Amherst, Amherst MA USA \\ ${ }^{2}$ Department of Chemical Engineering, University of Massachusetts Amherst, Amherst MA USA
}

Supporting Information Placeholder

\begin{abstract}
To produce next-generation, shelf-stable biosensors for point-of-care diagnostics, a combination of rugged biomolecular recognition elements, efficient encapsulants and innocuous deposition approaches are needed. Furthermore, to ensure that the sensitivity and specificity that is inherent to biological recognition elements is maintained in solid-state biosensing systems, site-specific immobilization chemistries must be invoked such that the function of the biomolecule remains unperturbed. In this work, we present a widely-applicable strategy to develop robust solid-state biosensors using emergent nanobody $(\mathrm{Nb})$ recognition elements coupled with a vapor-deposited polymer encapsulation layer. As compared to conventional immunoglobulin $\mathrm{G}$ ( $\mathrm{IgG}$ ) antibodies, Nbs are smaller (12-15 $\mathrm{kDa}$ as opposed to $\sim 150 \mathrm{kDa}$ ), have higher thermal stability and $\mathrm{pH}$ tolerance, boast greater ease of recombinant production, and are capable of binding antigens with high affinity and specificity. Photoinitiated chemical vapor deposition (piCVD) affords thin, protective polymer barrier layers over immobilized $\mathrm{Nb}$ arrays that allow for retention of $\mathrm{Nb}$ activity and specificity after both storage under ambient conditions and complete desiccation. Most importantly, we also demonstrate that vapor-deposited polymer encapsulation of nanobody arrays enables specific detection of target proteins in complex heterogenous samples, such as unpurified cell lysate, which is otherwise challenging to achieve with bare $\mathrm{Nb}$ arrays.
\end{abstract}

\section{INTRODUCTION}

Surface-immobilized proteins, antibodies and other similar biomolecules form the basis of most known biosensors, biomolecular electronics, and nanodevices. ${ }^{1-5}$ Although a number of surface immobilization chemistries are reported, comparatively fewer strategies for maintaining the function and stability of these surfaceimmobilized biomolecules, especially upon long-term storage, are known. ${ }^{6}$ Particularly for biosensors, it is imperative to maintain constant reaction conditions and to suppress nonspecific binding interactions that could lead to decreased target diffusion to the sensing interface and result in significant signal loss. ${ }^{7-8}$ Careless choice of surface treatments can damage biomolecules involved in the sensing process and, eventually, significantly shorten the shelf-life and accuracy of the biosensors over time. Therefore, special emphasis has been placed on encapsulation techniques, as the resultant surface barrier can simultaneously suppress sensor fouling due to nonspecific binding and/or adhesion, and produce a stable inner environment with optimal humidity and temperature for the biological sensing element. ${ }^{9-10}$

Typically, two kinds of polymer encapsulants are invoked for biosensors: hydrophilic polymers (or hydrogels), and zwitterionic polymers. ${ }^{11-14}$ These encapsulants are widely applied to form hydration layers at the sensor surface via either hydrogen bonds or ion solvation, respectively. Since the interaction force between water molecules or ions and the coating materials cannot be readily compensated by extra adsorption, this tightly bound water or ion layer becomes a physical, as well as an energetic, barrier against nonspecific adsorption of interferents. ${ }^{15}$ An emerging encapsulation strategy is to coat surfaces with polymers that present low surface energies, such as hyperbranched fluoropolymers, polypeptides, or silicone elastomers, and, thus, facilitate removal of adsorbed biomacromolecules by a limited shear force, like gentle rinsing. ${ }^{16-17}$

Layer-by-layer assemblies ${ }^{18-19}$ and in situ polymerized coatings $^{6,} 20$ also serve as encapsulants for intact biosensing systems. However, the non-conformal nature of these solution-processed coatings often hinders analyte diffusion to the underlying sensing element, which leads to inaccurate signal acquisition and increases signal integration times. Most critically, these methods require solvent usage, which is considered undesirable for reliably developing encapsulation layers on fragile biosensing elements, particularly proteins and antibodies 
that can degrade, denature or adopt inactive conformations depending on solvent exposure and variations in $\mathrm{pH}$ or ionic strength.

To produce next-generation, shelf-stable biosensors for point-of-care diagnostics, a combination of rugged biomolecular recognition elements, efficient encapsulants and innocuous deposition approaches are needed. Furthermore, to ensure that the sensitivity and specificity that is inherent to biological recognition elements is maintained in solid-state biosensing systems, site-specific immobilization chemistries must be invoked such that the function of the biomolecule remains unperturbed. In this work, we present a widely-applicable strategy to develop robust solid-state biosensors using a uniquely-stable nanobody $(\mathrm{Nb})$ recognition element coupled with a vapor-deposited polymer encapsulation layer.

\section{Experimental}

Materials.

(3-aminopropyl)trimethoxysilane (APTMS), 6-chlorohexanoic acid (CHA), N-(3dimethylaminopropyl)- $N$ '-ethylcarbodiimide

hydrochloride (EDC), dichloromethane (DCM), ethanol, 2-hydroxy-2-methylpropiophenone (HMPP), 2hydroxyethyl acrylate (HEA), $1 \mathrm{H}, 1 \mathrm{H}, 2 \mathrm{H}, 2 \mathrm{H}$ perfluorooctyl acrylate (TFOA), and 1,1,1,3,3,3hexafluoroisopropanol (HFIP) from MilliporeSigma and TCI America were used without purification.

Fabrication protocols. Clean Si wafers were immersed in APTMS 2\% in ethanol/water (90/10 v/v\%) solution for $5 \mathrm{~min}$. The treated wafers were washed with pure ethanol two times and further treated in an ultrasonic bath for 5 min to remove residual silanes on the surface. The washed substrates were blown dry using compressed $\mathrm{N}_{2}$ gas. For the introduction of chloroalkane group, $1.92 \mathrm{mg}$ of EDC $(0.01 \mathrm{mmol})$ and $1.50 \mathrm{mg}$ of 6-chlorohexanoic acid (0.01 $\mathrm{mmol}$ ) were added in $2 \mathrm{~mL}$ of DCM. Then each substrate was immersed in the solution for $12 \mathrm{~h}$. The grafted samples were further washed with pure DCM two times and then dried with $\mathrm{N}_{2}$ gas.

Protein expression and purification. Halo tagged Nanobody (Nb) constructs were expressed in BL21(DE3) pLysS E.coli cells in LB media supplemented with kanamycin $(25 \mu \mathrm{g} / \mathrm{mL})$ at $37{ }^{\circ} \mathrm{C}$ to $\mathrm{OD} 600 \sim 1.0$ and induced with $300 \mu \mathrm{M}$ IPTG at $16{ }^{\circ} \mathrm{C}$ for $16 \mathrm{~h}$. Cells were harvested by centrifugation at $5,000 \mathrm{~g}$ for $15 \mathrm{~min}$. The resulting cell pellets were resuspended in lysis buffer (50 $\mathrm{mM}$ Tris- $\mathrm{HCl} \mathrm{pH} \mathrm{8.0,300} \mathrm{mM} \mathrm{NaCl}$ and $1 \mathrm{mM}$ TCEP), lysed by sonication, and clarified by centrifugation at $30,000 \mathrm{~g}$ for $30 \mathrm{~min}$. Clarified lysate was incubated with Ni-NTA resin for $2 \mathrm{~h}$ at $4{ }^{\circ} \mathrm{C}$, washed with binding buffer (lysis buffer plus $10 \mathrm{mM}$ imidazole), and eluted into NiNTA elution buffer (lysis buffer plus $300 \mathrm{mM}$ imidazole). Then eluate was buffer exchanged into gel filtration buffer (50 mM Tris-HCl pH 8.0, $300 \mathrm{mM} \mathrm{NaCl}$ and $1 \mathrm{mM}$ DTT), concentrated and run on a Superdex 75 (GE) gel filtration column in gel filtration buffer at $0.3 \mathrm{~mL} / \mathrm{min}$. Recombinant UCH37 was expressed and purified as previously described. ${ }^{21}$

Cell lysate generation. Wild-type and UCH37 knockout (UCH37 ${ }^{\mathrm{KO}}$ ) HEK293 cells stably expressing RPN11-HTBH were grown, harvested and lysed in Ptsm lysis buffer (40 mM HEPES pH7.4, $40 \mathrm{mM} \mathrm{NaCl}, 10 \mathrm{mM}$ $\mathrm{MgCl}_{2}, 2 \mathrm{mM}$ ATP, $1 \mathrm{mM}$ DTT, and $10 \%$ glycerol). ${ }^{21}$ The lysates were clarified at $20,000 \mathrm{~g}$ for $20 \mathrm{~min}$ and the supernatant was collected and stored in $-80{ }^{\circ} \mathrm{C}$ prior to use. Concentration of total cell lysate was determined by BCA assay.

$\mathrm{Nb}$ immobilization. The grafted wafers were first washed with phosphate buffered saline (PBS) for $5 \mathrm{~min}$ at room temperature. Halo tagged control $\mathrm{Nb}$ and $\mathrm{Nb}$ were diluted to $5 \mu \mathrm{M}$ in cold PBS and incubated with washed wafers at $4{ }^{\circ} \mathrm{C}$ overnight with rocking. Then wafers were washed with PBS three times for $5 \mathrm{~min}$ to remove unreacted $\mathrm{Nb}$ and dried under air.

Protein target treatment and detection. Wafers with Halo tagged $\mathrm{Nb}$ immobilized were either treated with recombinant $\mathrm{UCH} 37$ or cell lysate directly or encapsulated first, followed by recombinant UCH37 or cell lysate treatment. Recombinant UCH37 was first diluted to $1 \mu \mathrm{M}$ in cold PBS and cell lysate was diluted to $1 \mathrm{mg} / \mathrm{mL}$ in cold PBS. Then the diluted UCH37 or cell lysate were incubated with wafers at $4{ }^{\circ} \mathrm{C}$ overnight with rocking. Then unbound UCH37 or total proteins from cell lysates were removed by washing wafers with PBS three times for $5 \mathrm{~min}$. To detect the presence of UCH37 on wafers, samples were treated with recombinant UCH37 antibody (Abcam, monoclonal Rabbit, 1:1000 dilution in TBS) at $4{ }^{\circ} \mathrm{C}$ overnight with rocking, followed by PBS wash three times and then treated with Goat anti-Rabbit IgG fluorescent secondary antibody (Licor, 1:15,000 dilution in TBS) at RT for 30 min with rocking. Then samples were washed three times with PBS for $5 \mathrm{~min}$ followed by brief sonication and dried under air. Fluorescent images were collected by scanning the wafer surface using a Licor Odyssey ${ }^{\circledR}$ CLx fluorescent imager (700 channel, $21 \mu \mathrm{m}$ ).

Polymer encapsulation. A custom-built reactor (stainless-steel walls, $290 \mathrm{~mm}$ diameter, $70 \mathrm{~mm}$ height) was used to polymerize HEA and TFOA directly on the surface of all samples using photoinitiated chemical vapor deposition (piCVD). A low-intensity UV lamp (UVP, UVLS-24 EL Series, 4Watt, $254 \mathrm{~nm}$ ) was turned on when the monomers were introduced into the reactor and the base pressure of the reactor had stabilized. During deposition, the base pressure of the reactor was kept at 
300 mTorr, while the stage temperature was maintained at $20{ }^{\circ} \mathrm{C}$ with a recirculating water cooling system. Photoinitiator HMPP and monomer (HEA or TFOA) were stored in glass ampules wrapped with fiberglass heating tape and introduced into the reactor through articulated needle valves, as illustrated in Figure S2. HMPP was heated to $110{ }^{\circ} \mathrm{C}$ and flowed into the reactor first for 5 min, then the monomer, also heated to $110{ }^{\circ} \mathrm{C}$, was introduced into the reactor, at which time polymerization and film deposition occurred. The deposition was allowed to proceed for $10 \mathrm{~min}$ to obtain an encapsulation layer. In the case of TFOA, $5 \mu \mathrm{L}$ of HFIP was dropcast onto the nanobody immobilized surface before deposition to promote TFOA wetting. After the deposition was completed, the samples were left on the substrate stage (maintained at $20{ }^{\circ} \mathrm{C}$ ) for a further $30 \mathrm{~min}$ until the temperature of reactants came to room temperature. This post annealing step allowed unreacted monomers to be pulled out of the film, which resulted in a porous coating on the substrate surface. The chemical structure and thickness of the piCVD film was determined by Fourier Transform Infrared (FTIR) Spectroscopy (Bruker Alpha) and atomic force microscopy (AFM) performed on films grown on bare glass or silicon substrates; equivalent thickness and polymer structure were assumed for encapsulation layers grown on nanobody arrays.

Surface characterization. Jupiter atomic force microscope (Asylum research, USA) was used to characterize the surface morphology of the samples. AFM images were obtained in tapping mode using PPP-NCHR probes (Nanosensors, Switzerland). The thickness of the samples was analyzed by spectroscopic ellipsometry (SE) using an RC2 Ellipsometer (JA Woollam, USA).

(a)

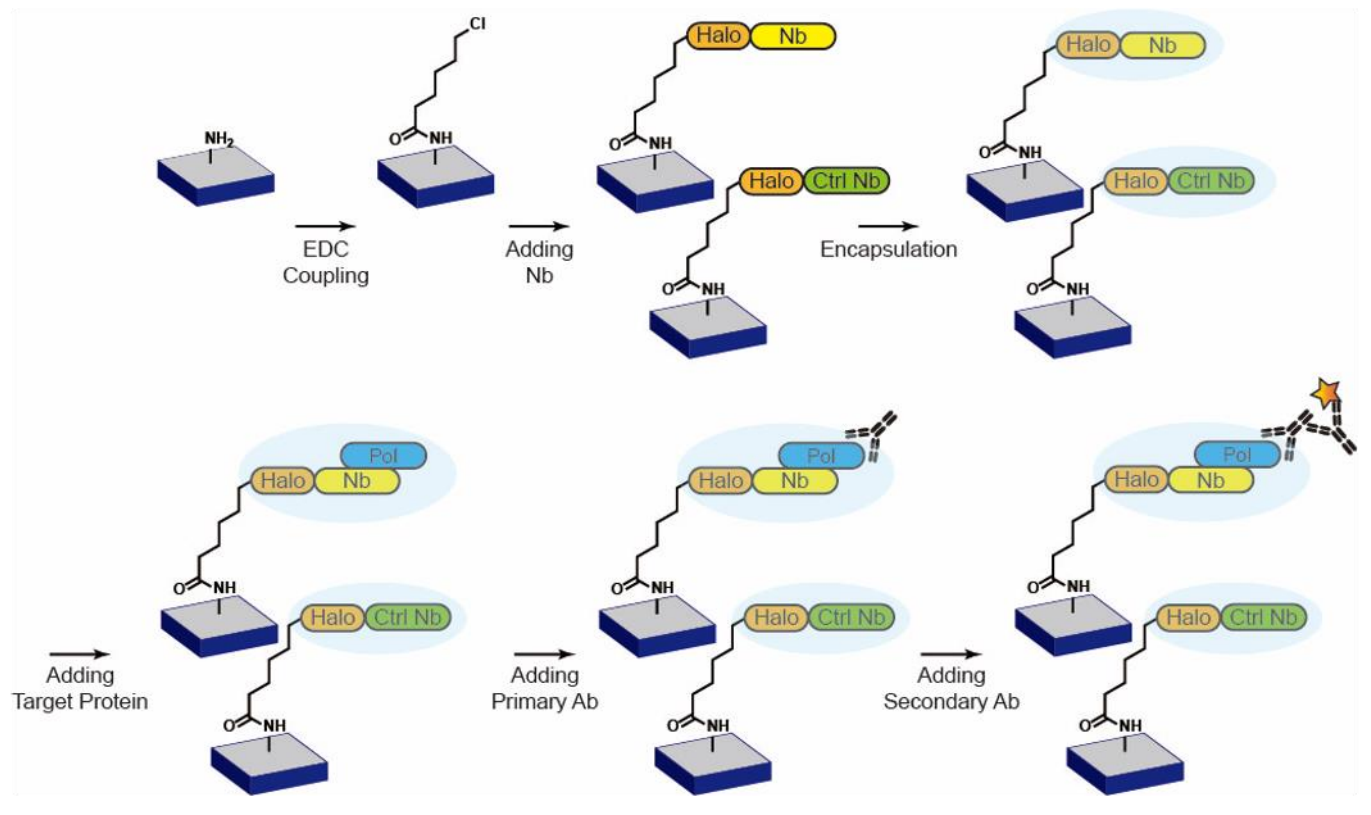

(b)

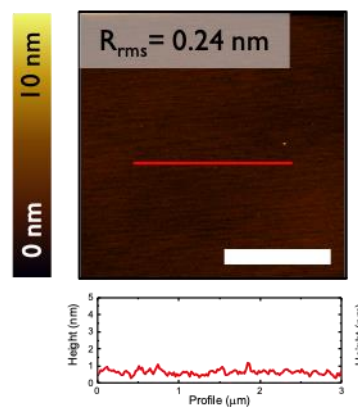

(c)
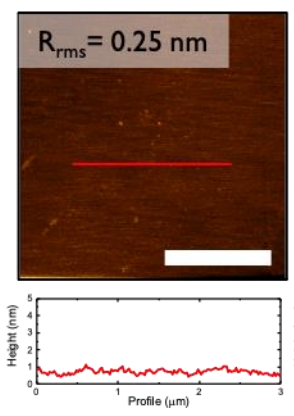

(d)
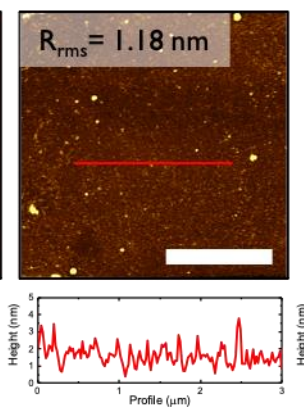

(e)
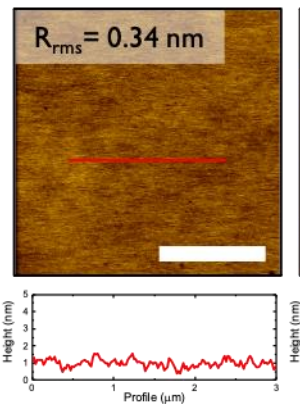

(f)

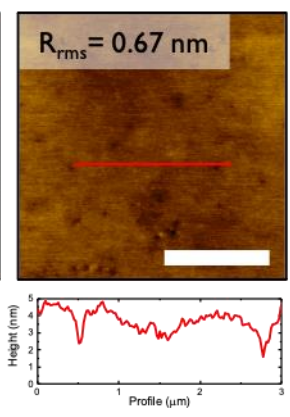

Figure 1. (a) Schematic illustration of the sensor fabrication process. (b-f) AFM topography images (top) and height profiles (bottom) of sequentially (b) ATPMS-treated, (c) CHA-treated, (d) Nb-treated, (e) pTFOA-encapsulated, and (f) target proteins/antibodies-exposed samples. 


\section{RESULTS AND DISCUSSION}

Nanobody Immobilization. Single variable domain (VHH) antibodies from camelids (referred to as nanobodies) have attracted a great deal of attention as biosensors. ${ }^{22-25}$ Similar to conventional immunoglobulin $\mathrm{G}$ (IgG) antibodies, nanobodies are capable of binding antigens with high affinity and specificity. What sets nanobodies apart from IgGs is their smaller size (12-15 $\mathrm{kDa}$ as opposed to $\sim 150 \mathrm{kDa}$ ), high thermal stability, $\mathrm{pH}$ tolerance, and ease of recombinant production. Due to these properties, we decided to build a robust, encapsulated sensing platform based on nanobodies. The nanobody (NbIII.15) chosen for these studies was derived from a synthetic yeast surface display library and engineered to bind with high affinity and specificity to the human protein UCHL5/UCH37 (LHC unpublished results).

Surface immobilization of proteins typically entails physical adsorption or covalent chemistry involving the canonical amino acid side chains, both of which provide little specificity in terms of the surface orientation. ${ }^{26}$ Thus, to avoid surface heterogeneity, we immobilized NbIII.15 on silane-treated silicon surface using the HaloTag system. ${ }^{27}$ As a control, a nanobody unable to bind the target protein $\mathrm{UCH} 37$ (NbCtrl) was also immobilized using the same approach. The entire fabrication process is illustrated in Figure 1a. At each step of the experiment, the thickness and the surface morphology of the samples were monitored by ellipsometry (Table S1) and AFM measurements (Figures $1 b, S 1)$, respectively.

Silanization with APTMS was readily achieved on silicon substrates. Within $5 \mathrm{~min}$, a smooth, amineterminated surface was isolated, as shown Figure 1b. EDC-coupling with 6-chlorohexanoic acid provided the chloroalkane moiety, necessary for covalent attachment to HaloTag. AFM topographic images (Figures 1b, c) and corresponding root mean square roughness (RMS) roughness $\left(\mathrm{R}_{\mathrm{rms}}\right)$ data show that smooth surfaces were obtained even after EDC coupling with 6-chlorohexanoic acid. HaloTag-fused nanobodies, NbIII.15 and NbCtrl, were then immobilized by covalent attachment to the chloroalkane linker. As summarized in Table S1, the thickness of the samples increased as the sensing layers were articulated. The resulting nanobody-decorated surfaces exhibit an increase in $\mathrm{R}_{\mathrm{rms}}$ of $0.9 \mathrm{~nm}$ and are uniform according to AFM topography (Figure 1).

Sensing and Binding Specificity of Immobilized Nanobody Arrays. To determine whether NbIII.15 retains function once immobilized, we monitored surface binding by fluorescence and SE. Fluorescence imaging reveals that recombinant $\mathrm{UCH} 37$ binds the surface decorated with NbIII.15, but not the surface with NbCtrl (Figure 2a). These images also confirm that fluorescent antibodies can be sequentially bound to the nanobodydecorated surface, further demonstrating that surfaceimmobilized nanobodies can provide a suitable sensing platform in the solid state.

(a)

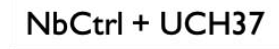

$\mathrm{Nb} I I I .15+\mathrm{UCH} 37$
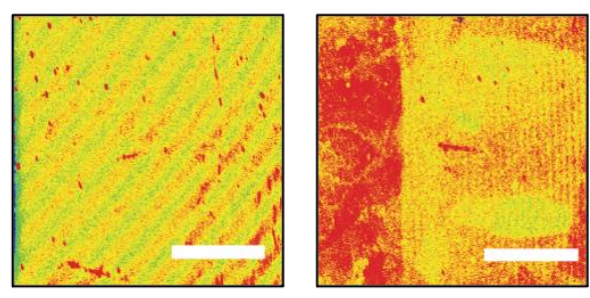

(b)

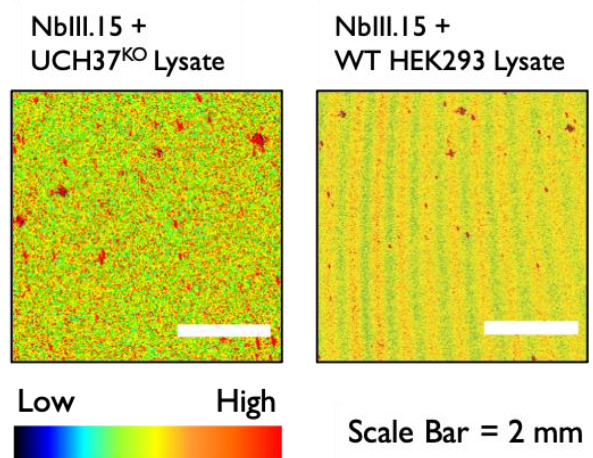

Figure 2. Fluorescence images of (a) purified UCH37treated samples on NbCtrl (left) and on the NbIII.15 arrays (right). (b) $\mathrm{UCH} 37^{\mathrm{KO}}$ lysate-treated (left) and HEK293 lysate-treated (right) samples on the NbIII.15 arrays.

The fluorescence microscopy images were also reinforced by SE; the thickness increases from 3.0 to 4.4 $\mathrm{nm}$ upon adding recombinant UCH37 to immobilized NbIII.15, but no change in thickness is observed when $\mathrm{UCH} 37$ is added to immobilized NbCtrl (Table S1). This confirms that the $\mathrm{Ctrl} \mathrm{Nb}$ has no binding affinity to the protein targets. In addition, the $\mathrm{R}_{\mathrm{rms}}$ of the surface increases from 0.95 to $1.46 \mathrm{~nm}$ (Figures $\mathrm{S} 1 \mathrm{c}$, d), indicating that the $\mathrm{Nb}$ has a stronger affinity towards target proteins.

While the results with purified recombinant UCH37 suggest that surface-bound NbIII.15 functions as predicted, a more stringent test requires the presentation of UCH37 in a complex, heterogenous mixture such as cell lysate. Thus, lysates derived from human embryonic kidney (HEK293) cells and UCH37-deficient HEK293 $\left(\mathrm{UCH} 37^{\mathrm{KO}}\right)$ cells were added to surfaces coated with either NbIII.15 or NbCtrl, and analyzed by fluorescence microscopy. The images show that, in contrast to the purified system, little binding occurs (Figure 2b). 
(a) PHEA encapsulated
$\mathrm{NbCtrl}+\mathrm{UCH} 37$

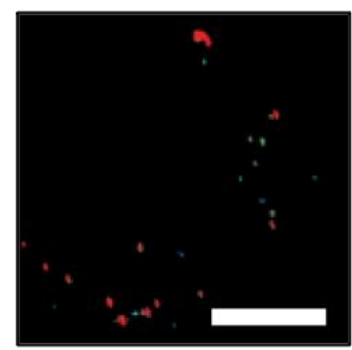

(c)
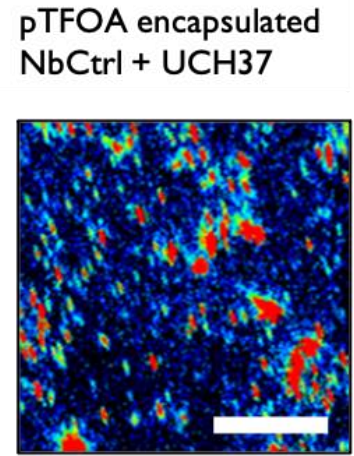

Low

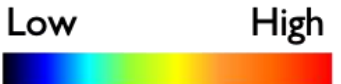

PHEA encapsulated

Nbll. 15 + UCH37

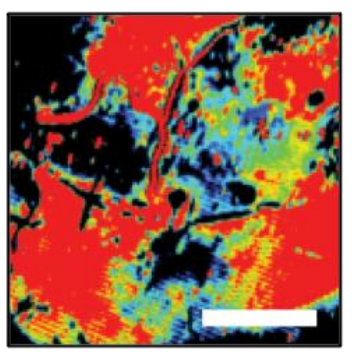

PTFOA encapsulated

NbllI.I 5 + UCH37

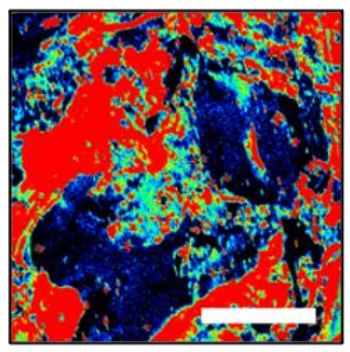

(b)

PHEA encapsulated

NbIll. 15 +

$\mathrm{UCH} 37^{\mathrm{KO}}$ Lysate

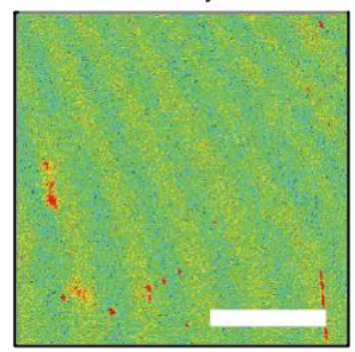

(d)

PTFOA encapsulated

Nblll. $15+$

$\mathrm{UCH} 37^{\mathrm{KO}}$ Lysate

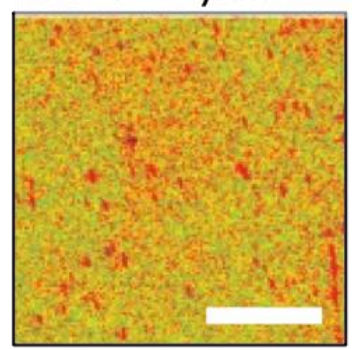

pHEA encapsulated

NbIII.I 5 +

WT HEK293 Lysate

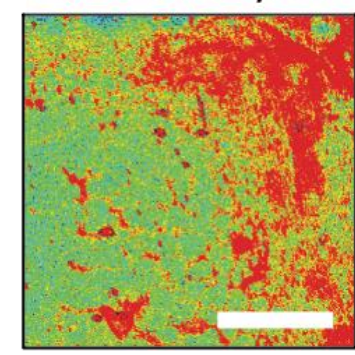

PTFOA encapsulated

NbIII.I 5 +

WT HEK293 Lysate

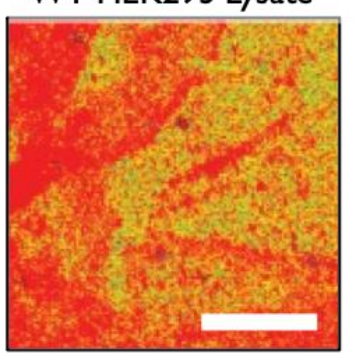

Figure 3. Fluorescence images of (a) purified UCH37-treated samples on pHEA-encapsulated NbCtrl (left) and on pHEAencapsulated NbIII.15 arrays (right). (b) UCH37 ${ }^{\mathrm{KO}}$ lysate-treated (left) and HEK293 lysate-treated (right) samples on pHEAencapsulated NbIII.15 arrays. (c) purified UCH37-treated samples on pTFOA-encapsulated NbCtrl (left) and on pHEAencapsulated NbIII.15 arrays (right). (b) UCH37 ${ }^{\mathrm{KO}}$ lysate-treated (left) and HEK293 lysate-treated (right) samples on pTFOAencapsulated NbIII.15 arrays. Scale bars are $2 \mathrm{~mm}$.

Encapsulation. We postulated that the lack of binding to the protein of interest in a complex, heterogenous environment was a result of sensor fouling due to nonspecific binding and/or interferent adhesion to the sensor surface. Thus, we decided to apply a mild, solventfree vapor deposition process to create a polymer barrier layer on the nanobody immobilized surface.

Chemical vapor deposition (CVD) is a solvent-free process in which vaporized precursors chemically react in the gas phase to generate a thin, solid film on a target surface. ${ }^{28-29} \mathrm{CVD}$ is also singularly able to produce films that are otherwise inaccessible via low-temperature solution-casting, such as fluoropolymer films and highlycrosslinked hydrophilic networks. Among several CVD methods to create polymer films, photo-initiated CVD (piCVD) is an especially mild iteration that does not require high-energy sources, high process temperatures or reactive initiators. ${ }^{30-31}$ Previous studies have demonstrated that specific protein-resistant hydrogels made of poly(2-hydroxyethyl methacrylate) (pHEMA) can be synthesized via piCVD on optical sensors without sacrificing sensitivity and accuracy. ${ }^{30}$ However, direct deposition of antifouling layers on surface-immobilized proteins and subsequent use of these sensing surfaces to screen for target analytes in complicated mixtures, such as cell lysate, have not been investigated.

We pursued the piCVD of two polymer encapsulants: a biocompatible hydrogel, poly(2-hydroxyethyl acrylate) (pHEA) and a fluoropolymer, poly $(1 \mathrm{H}, 1 \mathrm{H}, 2 \mathrm{H}, 2 \mathrm{H}$ perfluorooctyl acrylate) (pTFOA). Although the use of a superhydrophobic barrier layer for biosensors is counterintuitive, we posited that a microstructured or porous fluoropolymer encapsulant could mitigate undesired surface adhesion and fouling in complex sample fluids, such as cell lysates. Further, we hypothesized that the high intrinsic binding affinity of nanobodies to proteins of interest would drive target binding and signal generation, even if mass transport to the underlying nanobody recognition elements were attenuated by the pTFOA barrier layer. 
Under Vacuum

(a)

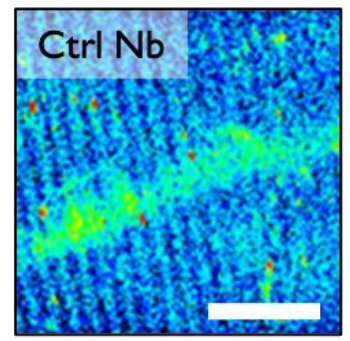

(c)

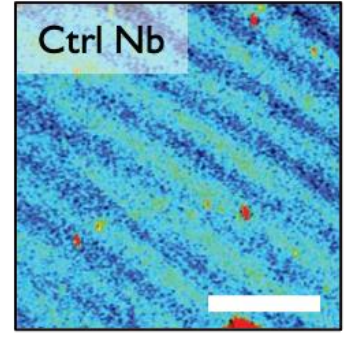

(e)

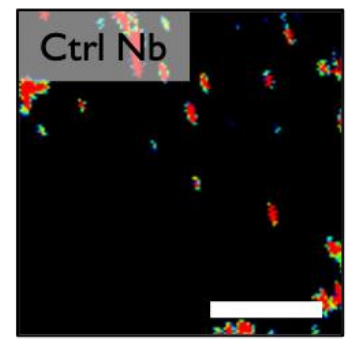

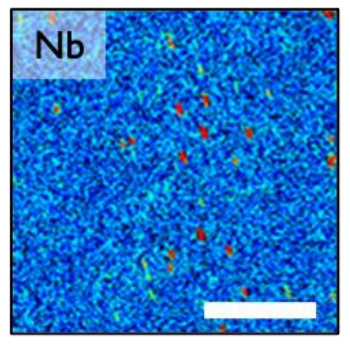
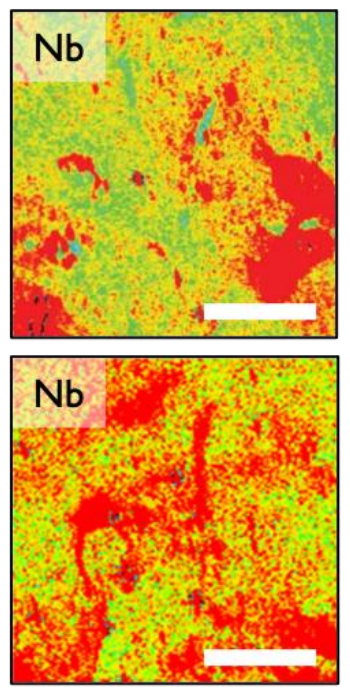

(b)

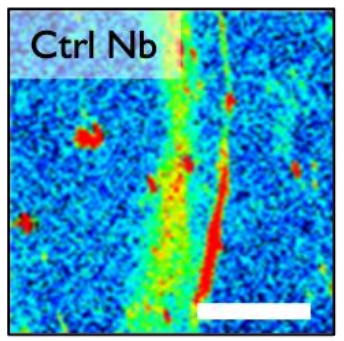

(d)

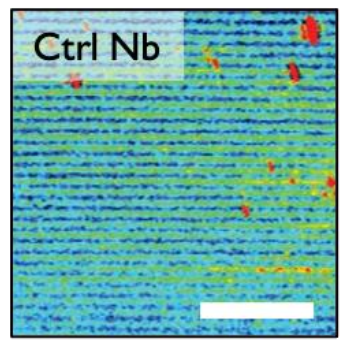

(f)

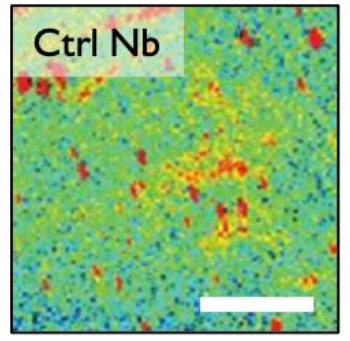

Under Air
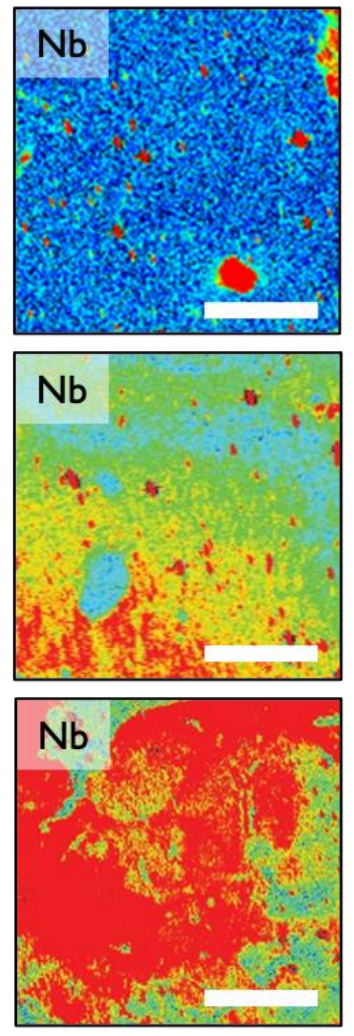

Low

High

Figure 4. Fluorescence images of HEK293 lysate-treated samples on NbCtrl (left) and Nb arrays (right). (a, c, e) samples kept under vacuum and (b, d, f) under desiccation: (a, b) samples without encapsulation, (c, d) pHEA-encapulsated samples, and (e, f) pTFOA-encapsulated samples. Scale bars are $2 \mathrm{~mm}$.

piCVD afforded uniform, nanometer-thick polymer coatings on the nanobody-decorated silicon surfaces. To reduce the time over which the nanobody arrays are subjected to vacuum conditions and UV light (which may cause photodegradation), we used a stepwise deposition algorithm to enact piCVD. Photoexcited carbonyl species are known to decompose into radicals from readily accessible triplet excited states, which can, in turn, initiate a free-radical polymerization. In our piCVD process, HMPP, the photoinitiator, was first introduced to the chamber followed by the monomer, HEA or TFOA, to effect polymerization. Under UV radiation of low intensity, HMPP decomposes and initializes the polymerization over the excited state lifetime of a carbonyl triplet state (ca. $100 \mathrm{~ns}$ ). As illustrated in Figure S3, the FTIR spectra of the HEA and pHEA showed a broad peak at $3200-3600 \mathrm{~cm}^{-1}$ corresponding to the hydroxyl group peak, and a sharp peak at $1720 \mathrm{~cm}^{-1}$, corresponding to the carbonyl stretching. Significantly,
pHEA did not exhibit a peak located at $1637 \mathrm{~cm}^{-1}$, where the stretching of an unsaturated $\mathrm{C}=\mathrm{C}$ double bond of HEA monomer should appear, thus confirming that the starting acrylate monomer was polymerized through the piCVD process. Likewise, FTIR spectra of TFOA and pTFOA also showed that TFOA was polymerized with HMPP using piCVD. In addition, residual monomers could have also been removed from the films during the postdeposition vacuum annealing step.

AFM topography images confirmed that uniform coatings were obtained over the entire nanobodydecorated surface (images for a pTFOA barrier layer are shown in Figure 1e, S4), without apparent defects. Moreover, the starting surface morphology of nanobodydecorated surfaces were not noticeably altered after polymer coating, tentatively suggesting that the dry piCVD process did not perturb the nanobody arrays. 
Sensing and Binding Specificity of Encapsulated Arrays. Next, we confirmed that the nanobody arrays retained their function after being encapsulated using piCVD. After encapsulation with pHEA, the $\mathrm{Nb}$ immobilized sample still displayed very high fluorescence signals, while the $\mathrm{Ctrl} \mathrm{Nb}$ did not yield a notable fluorescence signal (Figure 3a), indicating that the mild piCVD process does not interfere with the function or specificity of the $\mathrm{Nb}$ arrays. Similar behavior was also observed for nanobody arrays encapsulated with pTFOA (Figure 3c), confirming that this fluoropolymer barrier layer does not inhibit mass transport of target proteins. We posit that the pTFOA films created via piCVD enables the diffusion of target proteins to the underlying nanobody due to its discontinuous, wrinkled morphology, as shown in Figure S5, which originates from small gaps between monomer nucleation sites during the piCVD process.

The utility of the polymer barrier layers became evident when the nanobody arrays were presented with cell lysates. As opposed to the bare nanobody arrays, which did not demonstrate target binding in these complex, heterogenous matrices, pHEA and pTFOA encapsulated nanobody arrays readily displayed selective target binding (Figures 3b, d). The fluorescence obtained from lysate-treated $\mathrm{pHEA} / \mathrm{Nb}$ demonstrated that the pHEA encapsulation was able to reduce inference while maintaining target mass transfer toward the nanobody platform (Figure 3b). The effect of the encapsulation layer becomes stronger with pTFOA. As shown in Figure 3d, lysate-treated pTFOA/Nb sample shows higher fluorescence signals compared to other lysate-treated samples in Figure $3 b$. This demonstrates that pTFOA film most effectively mitigates undesired nonspecific binding without hampering the activity of the underlying $\mathrm{Nb}$.

Stability of Arrays After Storage and Desiccation. The stability of the $\mathrm{Nb}$ arrays were investigated under harsh environments in order to probe the efficacy of vapordeposited polymer encapsulants in protecting biological recognition elements. We first investigated the stability of bare, unencapsulated $\mathrm{Nb}$ arrays by placing them under vacuum or storing them in air overnight at room temperature. As shown in Figure 4a, b, the bare nanobody arrays became inactive after both desiccation and storing in air overnight. This highlights the need for a protective barrier. In contrast, both pHEA and pTFOA encapsulated $\mathrm{Nb}$ arrays retained their target binding activity after being exposed to air and vacuum (desiccation) storage conditions for more than $12 \mathrm{~h}$. Based on the low deviation in fluorescence signals observed for air and vacuum treated samples, we infer that the morphology and surface chemistry of these vapor-deposited encapsulation layers were not notably altered in these harsh storage conditions.
Moreover, the stronger fluorescence signal observed for pTFOA encapsulated samples indicated that a larger number of target binding events occurred in these samples, even after complete desiccation, as compared to the pHEA encapsulated samples. Therefore, we tentatively concluded that pTFOA is a strong candidate for biosensor encapsulation.

\section{CONCLUSION}

Here we demonstrate that vapor-deposited polymer encapsulation of immobilized nanobody arrays enables specific detection of target proteins in complex heterogenous samples. The nanobody chosen for this study was based on availability, but the site-specific, HaloTag immobilization approach and subsequent encapsulation is generalizable to any nanobody and corresponding target protein of interest. The key advance is the use of piCVD to create a polymer matrix that both protects the integrity of the nanobody array and allows for target protein diffusion. Future studies will focus on implementing this strategy to rapidly sense the presence of pathogens.

\section{ASSOCIATED CONTENT}

Supporting Information. AFM Images and spectroscopic ellipsometry data. The Supporting Information is available free of charge on the ACS Publications website.

\section{AUTHOR INFORMATION}

\section{Corresponding Author}

tandrew@umass.edu

estrieter@chem.umass.edu

\section{Author Contributions}

$\ddagger$ These authors contributed equally.

\section{Funding Sources}

No competing financial interests have been declared.

\section{ACKNOWLEDGMENT}

This material is based on work partially supported by the National Science Foundation under CBET 1706633 and the National Institutes of Health under R01GM1 10543.

\section{REFERENCES}

(1) Perumal, V.; Hashim, U. Advances in biosensors: Principle, architecture and applications. Journal of Applied Biomedicine 2014, 12 (1), 1-15.

(2) Limberis, L.; Magda, J. J.; Stewart, R. J. Polarized Alignment and Surface Immobilization of Microtubules for Kinesin-Powered Nanodevices. Nano Letters 2001, 1 (5), 277-280. 
(3) Skottrup, P. D.; Nicolaisen, M.; Justesen, A. F. Towards on-site pathogen detection using antibody-based sensors. Biosens. Bioelectron. 2008, 24 (3), 339-348.

(4) Steen Redeker, E.; Ta, D. T.; Cortens, D.; Billen, B.; Guedens, W.; Adriaensens, P. Protein Engineering For Directed Immobilization. Bioconjugate Chemistry 2013, 24 (11), 1761-1777.

(5) Yoon, J.; Shin, M.; Lee, T.; Choi, J.-W. Highly sensitive biosensors based on biomolecules and functional nanomaterials depending on the types of nanomaterials: A perspective review. Materials 2020, 13 (2), 299.

(6) Yang, Q. R.; Wu, B. C.; Eles, J. R.; Vazquez, A. L.; Kozai, T. D. Y.; Cui, X. T. Zwitterionic Polymer Coating Suppresses Microglial Encapsulation to Neural Implants In Vitro and In Vivo. Adv. Biosyst. 2020, 4 (6), 14.

(7) Lichtenberg, J. Y.; Ling, Y.; Kim, S. Non-specific adsorption reduction methods in biosensing. Sensors 2019 , 19 (11), 2488.

(8) Contreras-Naranjo, J. E.; Aguilar, O. Suppressing nonspecific binding of proteins onto electrode surfaces in the development of electrochemical immunosensors. Biosensors 2019, 9 (1), 15.

(9) Campuzano, S.; Pedrero, M.; Yáñez-Sedeño, P.; Pingarrón, J. M. Antifouling (bio) materials for electrochemical (bio) sensing. International journal of molecular sciences 2019, 20 (2), 423.

(10) Jiang, C.; Wang, G.; Hein, R.; Liu, N.; Luo, X.; Davis, J. J. Antifouling strategies for selective in vitro and in vivo sensing. Chemical reviews 2020, 120 (8), 3852-3889.

(11) Zhang, L.; Cao, Z.; Bai, T.; Carr, L.; Ella-Menye, J.-R.; Irvin, C.; Ratner, B. D.; Jiang, S. Zwitterionic hydrogels implanted in mice resist the foreign-body reaction. Nature Biotechnology 2013, 31 (6), 553-556.

(12) Xu, J.; Lee, H. Anti-Biofouling Strategies for LongTerm Continuous Use of Implantable Biosensors. Chemosensors 2020, 8 (3), 66.

(13) Erfani, A.; Seaberg, J.; Aichele, C. P.; Ramsey, J. D. Interactions between Biomolecules and Zwitterionic Moieties: A Review. Biomacromolecules 2020, 21 (7), 2557-2573.

(14) Vaisocherová, H.; Brynda, E.; Homola, J. Functionalizable low-fouling coatings for label-free biosensing in complex biological media: advances and applications. Analytical and bioanalytical chemistry 2015, 407 (14), 3927-3953.

(15) Paschke, S.; Lienkamp, K. Polyzwitterions: from surface properties and bioactivity profiles to biomedical applications. ACS Applied Polymer Materials 2020, 2 (2), 129-151.

(16) Damodaran, V. B.; Murthy, N. S. Bio-inspired strategies for designing antifouling biomaterials. Biomaterials research 2016, 20 (1), 1-11.

(17) Li, Z.; Guo, Z. Bioinspired surfaces with wettability for antifouling application. Nanoscale 2019, 11 (47), 2263622663.
(18) Liu, T.; Wang, Y.; Zhong, W.; Li, B.; Mequanint, K.; Luo, G.; Xing, M. Biomedical Applications of Layer-byLayer Self- Assembly for Cell Encapsulation: Current Status and Future Perspectives. Advanced healthcare materials 2019, 8 (1), 1800939.

(19) Sakr, O. S.; Borchard, G. Encapsulation of enzymes in Layer-by-Layer (LbL) structures: latest advances and applications. Biomacromolecules 2013, 14 (7), 2117-2135.

(20) Wang, J.; Hui, N. Zwitterionic poly(carboxybetaine) functionalized conducting polymer polyaniline nanowires for the electrochemical detection of carcinoembryonic antigen in undiluted blood serum. Bioelectrochemistry 2019, 125, 90-96.

(21) Deol, K. K.; Crowe, S. O.; Du, J.; Bisbee, H. A. ; Guenette, R. G. ; Strieter, E. R. Proteasome-Bound UCH37/UCHL5 Debranches Ubiquitin Chains to Promote Degradation. Molecular Cell 2020, 80(5), 796-809.

(22) Nevoltris, D.; Lombard, B.; Dupuis, E.; Mathis, G.; Chames, P.; Baty, D. Conformational Nanobodies Reveal Tethered Epidermal Growth Factor Receptor Involved in EGFR/ErbB2 Predimers. ACS Nano 2015, 9 (2), 1388-1399. (23) Buchfellner, A.; Yurlova, L.; Nüske, S.; Scholz, A. M.; Bogner, J.; Ruf, B.; Zolghadr, K.; Drexler, S. E.; Drexler, G. A.; Girst, S. A new nanobody-based biosensor to study endogenous PARP1 in vitro and in live human cells. PLoS One 2016, 11 (3), e0151041.

(24) Irannejad, R.; Tomshine, J. C.; Tomshine, J. R.; Chevalier, M.; Mahoney, J. P.; Steyaert, J.; Rasmussen, S. G. F.; Sunahara, R. K.; El-Samad, H.; Huang, B.; von Zastrow, M. Conformational biosensors reveal GPCR signalling from endosomes. Nature 2013, 495 (7442), 534538.

(25) De Meyer, T.; Muyldermans, S.; Depicker, A. Nanobody-based products as research and diagnostic tools. Trends in biotechnology 2014, 32 (5), 263-270.

(26) Anderson, G. P.; Liu, J. L.; Shriver-Lake, L. C.; Zabetakis, D.; Sugiharto, V. A.; Chen, H.-W.; Lee, C.-R.; Defang, G. N.; Wu, S.-J. L.; Venkateswaran, N.; Goldman, E. R. Oriented Immobilization of Single-Domain Antibodies Using SpyTag/SpyCatcher Yields Improved Limits of Detection. Analytical Chemistry 2019, 91 (15), 9424-9429.

(27) Los, G. V.; Encell, L. P.; McDougall, M. G.; Hartzell, D. D.; Karassina, N.; Zimprich, C.; Wood, M. G.; Learish, R.; Ohana, R. F.; Urh, M. HaloTag: a novel protein labeling technology for cell imaging and protein analysis. ACS chemical biology 2008, 3 (6), 373-382.

(28) Bilger, D.; Homayounfar, S. Z.; Andrew, T. L. A critical review of reactive vapor deposition for conjugated polymer synthesis. Journal of Materials Chemistry C 2019, 7 (24), 7159-7174.

(29) Yagüe, J. L.; Gleason, K. K. Enhanced cross-linked density by annealing on fluorinated polymers synthesized via initiated chemical vapor deposition to prevent surface reconstruction. Macromolecules 2013, 46 (16), 6548-6554. (30) Baxamusa, S. H.; Montero, L.; Dubach, J. M.; Clark, H. A.; Borros, S.; Gleason, K. K. Protection of sensors for 
biological applications by photoinitiated chemical vapor deposition of hydrogel thin films. Biomacromolecules 2008, 9 (10), 2857-2862.

(31) Baxamusa, S. H.; Gleason, K. K. Random copolymer films with molecular-scale compositional heterogeneities that interfere with protein adsorption. Advanced Functional Materials 2009, 19 (21), 3489-3496. 